\title{
Model-Based FDIR: from paper to planes
}

\author{
Andrés Marcos*1 Luis F. Peñín** Augusto Caramagno***1 \\ *University of Bristol, Bristol, UK (Tel: +44 117954 5607; e-mail: andres.marcos@bristol.ac.uk) \\ ** Deimos Space SL, Madrid, Spain (e-mail: luis-felipe.penin @deimos-space.com) \\ *** Swiss Space Systems, Payerne, Switzerland
}

\begin{abstract}
Model-based fault detection, identification and reconfiguration (FDIR) methods rely on the idea of analytical redundancy in which, in contrast to physical or hardware redundancy, real physical measurements are complemented with analytically computed redundant variables. A standard method to analytically detect the existence of a failure is to look for anomalies in the plant's output relative to a model-based estimate of that output generating a signal called residual. If the generated residual includes enough information to precisely identify the fault, then it can be used to ameliorate the fault effects or help degrade the performance of the system in a controlled fashion. Model-based FDIR methods have been studied and matured in Academia during the last 20 years but in the realm of the aerospace domain they have not achieved the desired attention due to the critical nature of faults and the difficulty of testing, verifying and validating the resulting designs. In this paper, main efforts by Deimos Space to bring these methods to a mature stage for industrial utilization and deployment are reviewed with emphasis on $\mathrm{H}_{\infty}$ optimization FDI technology.
\end{abstract}

Keywords: Model-based fault detection isolation and reconfiguration, aerospace systems

\section{INTRODUCTION}

Fault detection, identification and reconfiguration (FDIR) approaches based on mathematical models, the so-called model-based methods, have been proposed and consolidated in Academia for the last 20 years [Chen and Patton 1999 Marzat et al 2012]. From an aerospace industrial control perspective these methods are well mastered and operationally implemented but from the perspective of FDI, and due to the criticality of testing fault scenarios in real aeronautical environments, most efforts until recently were very timid research and development studies.

Addressing this shortcoming, the last few years have seen concerted efforts at the European level, both in Aeronautics as well as Space, to change this situation. This article presents a summary of several aeronautical projects providing a time-line from theory up to operational deployment assessment for a model-based FDI approach based on the $\mathrm{H}_{\infty}$ formalism [Doyle et al 88, Appleby 90, Edelmayer et al 94, Balas et al 98].

All the presented cases, except for the $1^{\text {st }}$ case in Section 4.1, were performed by Deimos Space as part of a strategy to mature and consolidate FDI technology in their technical portfolio. It is noted that although in here only the aeronautical component is described, these efforts were intertwined with similar projects in the Space side supported by the European Space Agency.

The layout of the paper is as follows. Section 2 cursorily describes model-based FDI approaches in general and the $\mathrm{H}_{\infty}$
FDI approach in particular. Section 3 describes the increasing Technological Readiness Level (TRL) application of the latter to different aircraft, from a basic level (TRL 2) in a Boeing 747-100/200 simulation model, to verification and validation by Airbus on their state-or-the-art test benches (TRL 5/6) for assessment of the potential for subsequent operational maturation and deployment.

\section{MODEL-BASED APPROACHES: (FROM) THEORY}

In this section the main ideas of model-based FDI are presented followed by a methodological sketch of $\mathrm{H}_{\infty}$ FDI design. This should provide a basic understanding of the methods and represents the "paper" aspect of the title.

\subsection{Model-Based Approaches}

The essence of model-based fault detection and isolation (FDI), or fault detection and diagnosis (FDD), problem is depicted in Fig.1 and can be formalized as follows -assuming there are no other disturbances besides the fault nor uncertainty:

FDI/FDD problem: Given a model of the nominal system $G_{u}$ and knowledge (measured or estimated) of the inputs $u$ and outputs $y$ of the system, determine a filter $F=\left[F_{u} F_{y}\right]^{T}$ that provides a fault estimate res $=\hat{f}$ with information on the faults fentering the system (through $G_{f}$ ).

The fault estimate res $=\hat{f}$ can provide just an indication of the fault's presence (fault detection) or also the fault location and

\footnotetext{
${ }^{1}$ A. Marcos and A. Caramagno were at Deimos Space SL during the course of the work presented in this paper.
} 
source (fault isolation) [Chen and Patton 99, Marzat et al 12]. Fault detection requires only a single (scalar) value while fault isolation requires a set (vector) of fault estimates in order to be able to distinguish between different faults.

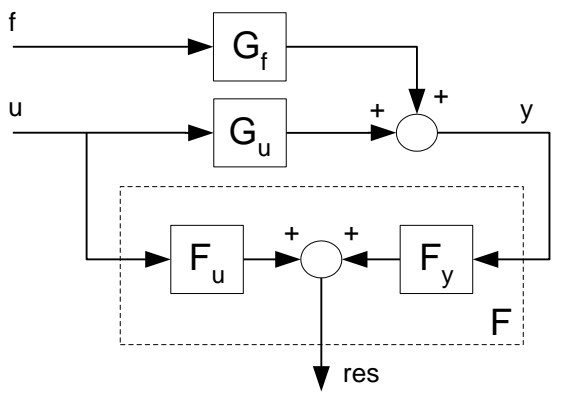

Fig.1 Basic model-based FDI design architecture

From the above figure it is straight forward to obtain:

$$
\begin{aligned}
r e s & =F_{y} y+F_{u} u=F_{y}\left(G_{f} f+G_{u} u\right)+F_{u} u \\
& =F_{y} G_{f} f+\left(F_{u}+F_{y} G_{u}\right) u
\end{aligned}
$$

From where the main objectives in the design of an FDI/FDD filter are easily identified:

1. Maximize the effect of the faults (i.e. $F_{y}$ should maximize the effects of the faults $f$ based on the knowledge of their effect on the system, $G_{f}$ ).

2. Minimize any other effect (e.g. controller input $u$, uncertainty, disturbances, other subsystem faults...).

Note that in the present case, the filter can be optimally designed by setting $F_{y}=G_{f}^{-1}$ and $F_{u}=-F_{y} G_{u}$. In the general case, the two filter components must be traded-off to fulfil the two competing objectives. For example, accounting for external disturbances effects on the system (ie adding $G_{d} d$ to the output of the system $y$ ), Equation 1 transforms into:

$$
\text { res }=F_{y} G_{f} f+F_{y} G_{d} d+\left(F_{u}+F_{y} G_{u}\right) u
$$

This clearly shows that if a fault affects the system along the same direction as a disturbance (i.e. $G_{f}=G_{d}$ ) then it is not possible to detect such fault. In the case of uncertainty, the analysis is similar but leads to a more complicated trade-off.

The above discussion was based on an open-loop FDI design perspective (i.e. not including the controller), although it is also possible to perform FDI on closed-loop system. Openloop FDI is favoured, see [Niemann and Stoustrup 97], essentially due to its ability to use more information on the system (i.e. the presence of the controller hides information by absorbing the fault effects due to its natural robustness characteristics) as well as due to its ability to retrofit the designed FDI filter on an existing controlled system. For both type of approaches, the formal requirements are the same:

1. To reliably and accurately detect and isolate faults

2. To be insensitive to exogenous disturbances, noise and system uncertainty

\subsection{H-infinity FDIR}

The above objectives lead to the natural requirement that the residual generator be robust, which is the main motivation for the use of $\mathrm{H}_{\infty}$ optimization FDI approaches since one of their distinguishing features is the explicit inclusion of uncertainty [Doyle et al 88, Balas et al 91, Chen and Patton 99]. As such, they provide for a direct way to trade-off the level of robustness to uncertainty with the level of performance of the FDI filter. This is especially important in filtering problems, since it has a direct impact on the false alarm versus missed fault rates -which are critical for the practical applicability of an FDI filter and its industrial deployment.

The problem of designing a robust FDI filter is generally divided in two main stages: robust residual generation and robust residual evaluation. Within the task of residual generation, and from the $\mathrm{H}_{\infty}$ perspective, the idea is to obtain a filter $F=\left[F_{u} F_{y}\right]^{T}$ so that $F_{u}$ cancels the effects of the known inputs and $F_{y}$ maximizes the effects of the fault while minimizing the effects of the disturbances based on the frequency regions in which they act. This is accomplished through the use of so-called weighting filters $W_{*}$ which embed the knowledge on these different frequency regions as well as the desired design objectives into the $\mathrm{H}_{\infty}$ optimization framework. A methodology used for the design of an optimal FDI filter is as follows [Appleby 90, Mangoubi 98, Balas et al 91, Marcos et al 05]:

1. Obtain the linear time invariant (LTI) model that will be used for design, i.e. find $G=\left[G_{f} G_{d} G_{u}\right]^{T}$. Typically, this is obtained by Taylor's $1^{\text {st }}$ order approximation of the nonlinear system around a specific point in the operation envelope.

2. Define the $\mathrm{H}_{\infty}$ FDI interconnection, which represents the posing of the mathematical optimization problem in similar fashion to Figure 1. It specifies the input and output channels, establishes the design rationale and includes the LTI system above as well as the optimization weights (see next point).

3. Define the optimization problem weights. These are used to map the performance and robustness design specifications into the mathematical interconnection. The general types and ideas behind weight definition are:

- Noise weights. They are used to attenuate the noise/disturbance effects on the residual. Typically high-pass weights are used to indicate the highfrequency noise associated with sensor systems.

- Ideal fault weights. Typically low-pass weights that contribute to shape the bandwidth and performance of the filter. If several faults are desired to be identified, then a diagonal matrix is used to emphasize their de-coupling on the residuals.

- Error/performance weights. These weights parallel the performance weights used in the standard control set-up. The idea is to minimize the error at low frequencies and relax the constraints at higher frequencies. Hence low-pass weights are typically selected for the actuator and sensor errors. 
4. Establish the performance metric. The performance metric for $\mathrm{H}_{\infty}$ synthesis is based on the induced $\mathrm{L}_{2}$-norm, e.g. the $\mathrm{H}_{\infty}$ norm between the selected input-to-output transfer functions.

5. Solve the optimization problem. The LTI $\mathrm{H}_{\infty}$ optimization approach finds the FDI filter design by exactly characterizing, and thus guaranteeing, the induced $\mathrm{L}_{2}$-norm performance of the weighted transfer functions established in the $\mathrm{H}_{\infty}$ interconnection. It does so by using a quadratic Lyapunov function and solvability conditions from the wellknown Bounded Real Lemma [Doyle et al 88].

In order to exemplify the methodology, assume that a nominal LTI plant $G=\left[G_{f} G_{d} G_{u}\right]^{T}$ is given and it is desired to design an FDI filter $F=\left[F_{u} F_{y}\right]^{T}$ following a design rationale based on fault model-matching (this has several advantages, see [Chen and Patton 1999, Marcos et al 05a]). First, the $\mathrm{H}_{\infty}$ FDI interconnection is established following this fault modelmatching rationale, see Figure 2. The proposed interconnection has three optimization weights: $W_{f}$ (shaping the ideal fault knowledge and/or desired filter behaviour), $W_{d}$ (shaping the disturbances) and $W_{u}$ (shaping the known controller command).

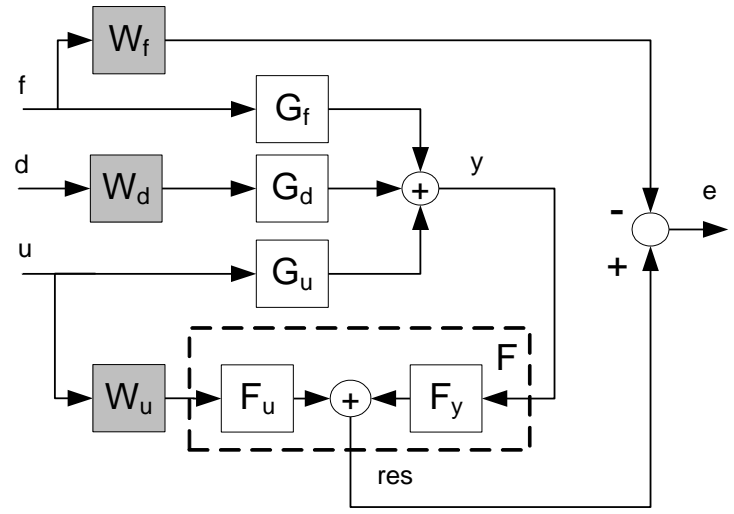

Fig.2 Ideal fault-matching FDI $\mathrm{H}_{\infty}$ interconnection

Based on steps 4 and 5, then the optimization will minimize the $\mathrm{L}_{2}$-norm of the transfer function (TF) from the inputs to the error: $\min \left\|T F_{[f d u] \rightarrow e}\right\|_{\infty}$. From algebraic manipulations, the following is obtained:

$$
\begin{aligned}
& e=r e s-W_{f} f \\
& =\left(F_{y} G_{f}-W_{f}\right) f+F_{y} G_{d} W_{d} d+\left(F_{y} G_{u}+F_{u} W_{u}\right) u
\end{aligned}
$$

Thus, three main TFs are obtained with the first one related to FDI performance (i.e. perfect identification if the TF given by $\left(F_{y} G_{f-} W_{f}\right)$ is zero), the second related to disturbance rejection and the last to known command cancellation. The shaping filters are used to emphasize the frequency regions where we want to achieve these objectives, resulting in a direct way to perform the design trade-offs. Solving the optimization problem will yield the desired $F=\left[\begin{array}{ll}F_{u} & F_{y}\end{array}\right]^{T}$ based on the defined interconnection (Fig.2), shaping weights $\left(W_{f}, W_{d}, W_{u}\right)$ and plant $\left(G_{f}, G_{d}, G_{u}\right)$.

\section{MODEL-BASED APPROACHES: (TO) PRACTICE}

In this section, several aeronautical cases are presented in chronological order and covering from the early part of the research to prove feasibility stage (TRL 2) up to system demonstration in relevant environment (TRL 6). As noted before, all except for the first case were performed at Deimos Space in the course of the last 7 years (2006-2013).

\subsection{High-Fidelity Simulation in a Boeing 747-100/200}

The case considered here [Marcos et al 05a] is to the best of the authors, and also from the detailed survey of [Marzat et al 12], the first instance that an $\mathrm{H}_{\infty}$ FDI filter was designed for a high-fidelity, full nonlinear aircraft model (although [Melody et al 01] showed first its applicability from the perspective of ice detection). Despite the basic technology research level (TRL 2, i.e. computer simulation) it served to pave the way to accept the plausibility of the approach for aircraft FDI, which as will be exemplified with the subsequent cases was correct.

The aircraft model used for this application was a Boeing 747 series 100-200, see Fig.3 [Hanke et al (1970, 1971)]. The focus of the application was on the longitudinal motion of the aircraft although the simulations were performed with the full nonlinear model. A movable horizontal stabilizer with four elevator segments (i.e. two inboards and two outboards) plus the thrust from four engines were used to control the longitudinal motion.

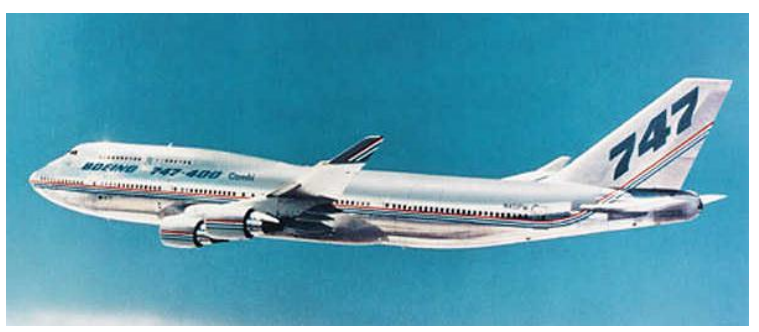

Fig. 3 Boeing 747-100/200 (courtesy of Boeing)

The $\mathrm{H}_{\infty}$ FDI design rationale was that of fault-matching with "tracking" (i.e. detection) requirements. The filter was designed to detect and isolate elevator actuator and pitch rate sensor faults while rejecting disturbances and noise. The paper covered: [1] selection of the plant for FDI design, [2] definition of optimization interconnection (Fig.4), [iii] weight design, and [3] frequency (Fig.5) and time (Fig.6) response analysis for filter tuning.

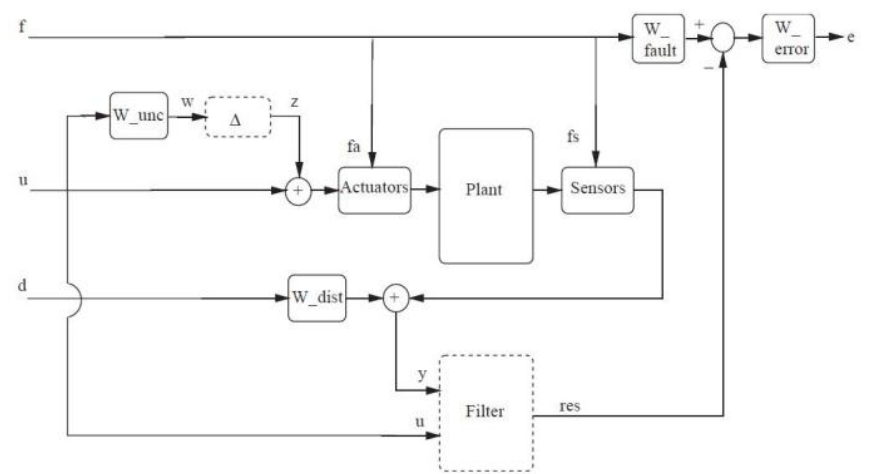

Fig. 4 Boeing $747 \mathrm{H} \infty \mathrm{FDI}$ design interconnection 

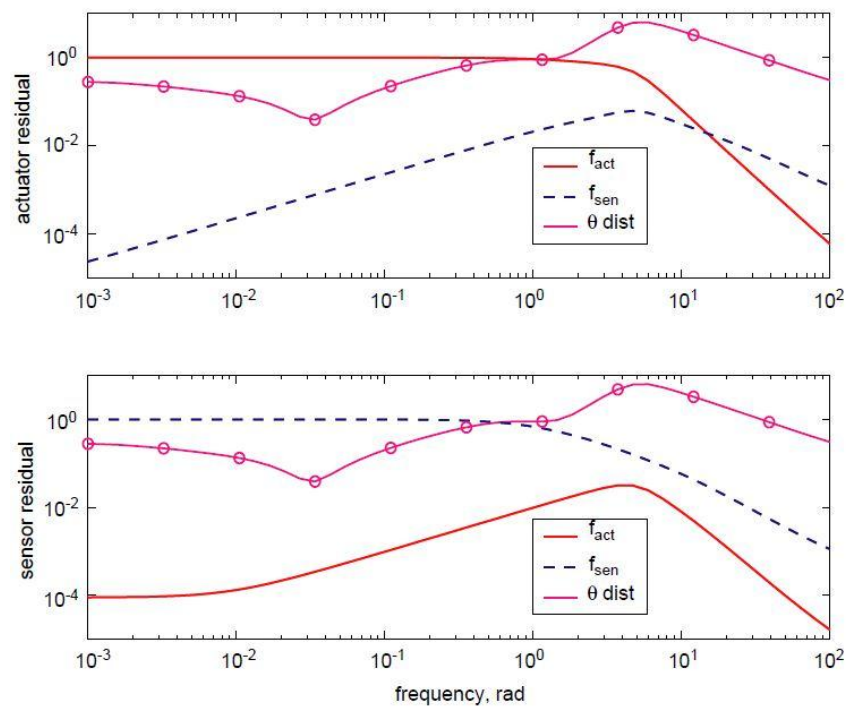

Fig. 5 Boeing 747: FDI transfer function analysis

The nonlinear time simulations (including sensor noise and gust) showed the good properties of the filters for detecting the fault despite changes in the aircraft arising from longitudinal manoeuvres, see Fig 6.
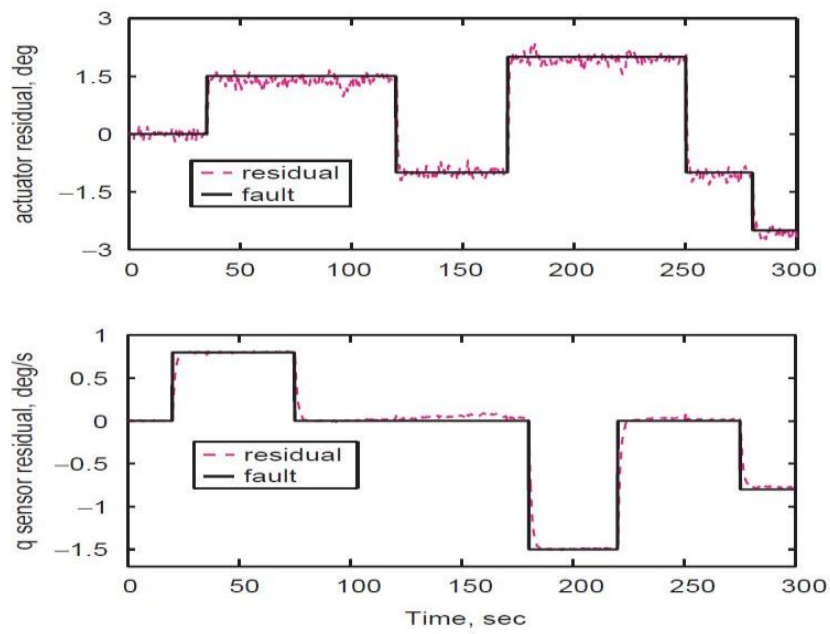

Fig. 6 Boeing 747: FDI nonlinear closed-loop response

The above developments were applied by Deimos to a sophisticated atmospheric re-entry vehicle simulation model based on EADS re-entry demonstrator Phoenix, see [Kerr et al 08], as well as to piloted simulations for a twin-engine research aircraft, see [Kerr et al 09]. The latter also including real flight test data assessment.

\subsection{EU-FP7 ADDSAFE Project}

A consortium of European industries (Airbus, Deimos Space), research centers (DLR, SZTAKI, IMS-CNRS) and Universities (Delft, Leicester, Hull) was established in July 2009 for three years to research model-based FDI methods for aircraft. The project, co-funded by the European $7^{\text {th }}$ Framework Program and led by Deimos Space, was entitled "Advanced Fault Diagnosis for Sustainable Flight Guidance and Control (ADDSAFE)" - see Fig7 and for further details the webpage of the project: http://addsafe.deimos-space.com/ and reference [Marcos 13].

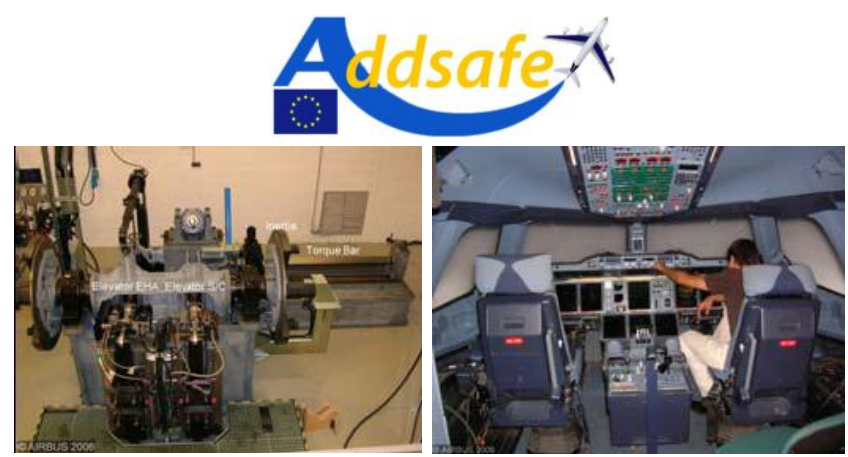

Fig. 7 ADDSAFE: Airbus test facilities (left) Elevator SystemIntegration-Bench and (right) Flight Simulator

The overall aim of ADDSAFE was to research and develop model-based FDI methods for aircraft flight control systems faults, predominantly sensor and actuator malfunctions. The thrust of the project was based on the link between aircraft sustainability and FDI. For example, it has been demonstrated that improving the fault diagnosis performance in flight control systems allows to optimize the aircraft structural design (resulting in weight saving), which in turn helps improve aircraft performance and to decrease its environmental footprint [Goupil 10]. The importance of the studies carried out within the project arise, on the one hand, due to the representativeness of the benchmark proposed by Airbus, which consisted of a generic civil aircraft model and realistic fault scenarios, and on the other hand, the industrial validation of the more promising designs in the actual Airbus flight control system Verification \& Validation $(\mathrm{V} \& \mathrm{~V})$ process [Goupil and Marcos 11].

The different design teams used model-based approaches covering geometric, nonlinear local, system identification, sliding-mode observers and $\mathrm{H}_{\infty}$ based filtering among others. Deimos' design [Marcos 12] was based on the latter and aimed at detection of faults in aileron actuators, see Fig 8.
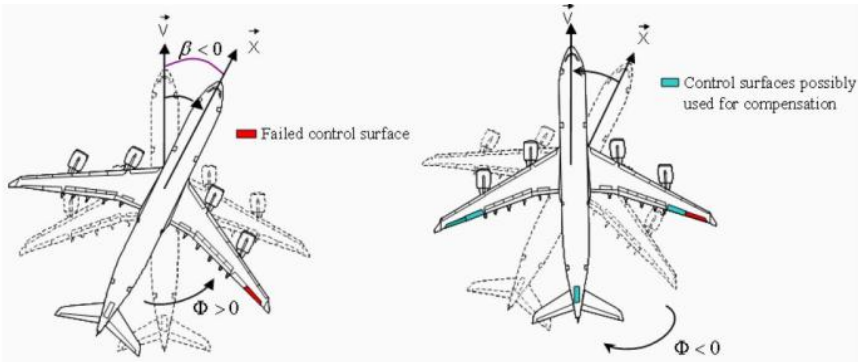

Fig.8 ADDSAFE: Effect of aileron jamming and potential control surfaces used for compensation

Deimos design followed a global aircraft FDI approach, i.e. using sensor measurements from the aircraft as opposed to only inputs/outputs of the actuator, for the $\mathrm{H}_{\infty}$ FDI filter (residual generation) and a simple time/magnitude logic (residual evaluation) for the fault isolation between left and right inboard ailerons. Fig.9 graphically shows the design process followed -which parallels Section 2.2 and also condenses in a more methodological fashion the steps in the previous example cases. 


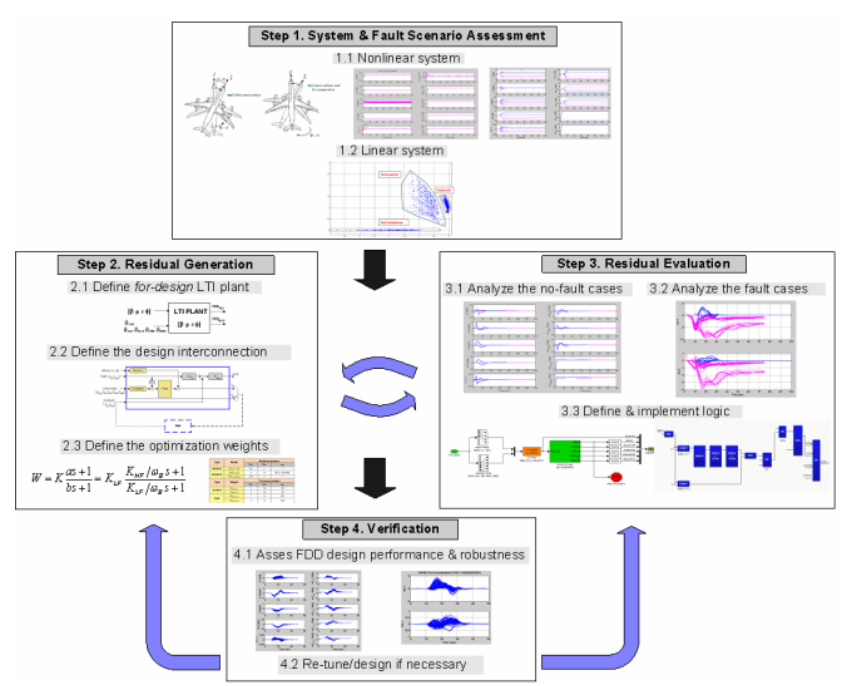

Fig.9 ADDSAFE: Deimos methodology for $\mathrm{H}^{\infty}$ FDI design

In order to facilitate the industrialization transfer of the methodology, didactic efforts such that of Fig.9 as well as standardization of the design weights' tuning were emphasized. With respect to the later, a generic first-order transfer function model was used -see Eq 4, where the lowfrequency gain is given by $K_{L F}=K$, the high-frequency gain by $K_{H F}=K a / b$ and the bandwidth by $\omega_{B}=K_{L F} / b=K_{H F} / a$ :

$$
W=K \frac{a s+1}{b s+1}=K_{L F} \frac{K_{H F} / \omega_{B} s+1}{K_{L F} / \omega_{B} s+1}
$$

An example of the performance of the design is given in Table 1. This table summarizes the main detection metrics (i.e. false alarm FA, missed-detection MD and normalized detection time performance DTP) arising from a parametric campaign using Airbus nonlinear computer simulation model and Deimos Functional Engineering Simulator (FES). The campaign used different flight conditions as defined by parameters such as altitude, speed, mass, center of gravity and inertia for several fault scenarios.

Table1 ADDSAFE: Deimos FDI metrics

\begin{tabular}{|c|c|c|c|c|c|c|c|}
\hline \multicolumn{3}{|c|}{ Info } & \multicolumn{2}{c|}{ Rates (\%) } & \multicolumn{3}{c|}{ Normalized DTP } \\
\hline \multirow{2}{*}{ Set } & \# runs & FA & MD & Min & Mean & Max \\
\hline \multirow{2}{*}{ TOTAL } & 3792 & 0 & 0 & 0.016 & 0.084 & 0.388 \\
\hline \multirow{2}{*}{ ALI } & S1.1 & 316 & 0 & 0 & 0.220 & 0.226 & 0.377 \\
\cline { 2 - 8 } & S1.2 & 316 & 0 & 0 & 0.061 & 0.211 & 0.264 \\
\cline { 2 - 8 } & S1.3 & 316 & 0 & 0 & 0.016 & 0.029 & 0.213 \\
\hline \multirow{2}{*}{ ARI } & $\mathbf{S 1 . 1}$ & 316 & 0 & 0 & 0.119 & 0.230 & 0.388 \\
\cline { 2 - 8 } & $\mathbf{S 1 . 2}$ & 316 & 0 & 0 & 0.174 & 0.212 & 0.272 \\
\cline { 2 - 8 } & $\mathbf{S 1 . 3}$ & 316 & 0 & 0 & 0.024 & 0.045 & 0.215 \\
\hline \multicolumn{2}{|c|}{ No fault } & 1896 & 0 & -- & -- & -- & - \\
\hline
\end{tabular}

Deimos design was one of the five (out of 13) selected by Airbus for industrial validation [Goupil and Marcos 012].

\subsection{EU-FP7 RECONFIGURE Project}

Sparked by the success of ADDSAFE, another EU FP7 project was initiated in January 2013 for another 3 years. This project, also under the leadership of Deimos Space and with participation of Airbus, DLR, SZTAKI, ONERA and the Universities of Exeter, Cambridge and Delft is entitled "Reconfiguration of Control in Flight for Integral Global Upset Recovery (RECONFIGURE)", see Fig.10.
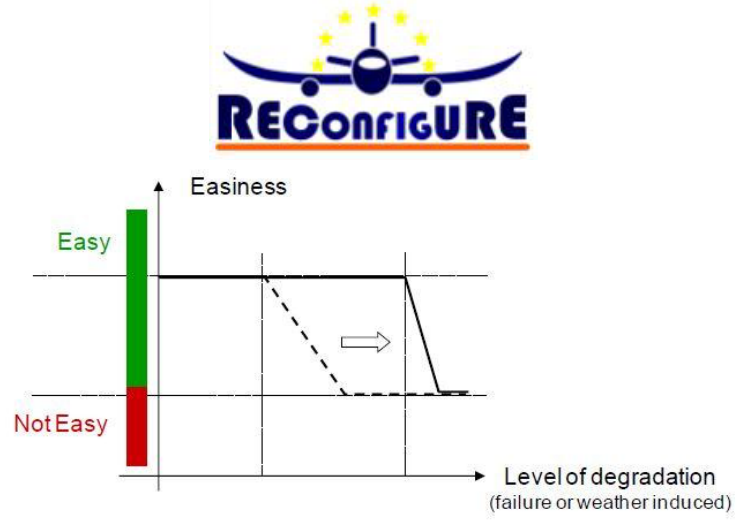

Fig.10 RECONFIGURE: project's goal

The main goal of RECONFIGURE is to investigate and develop aircraft guidance and control $(\mathrm{G} \& \mathrm{C})$ technologies that facilitate the automated handling of off-nominal and abnormal events optimizing the aircraft status and flight. This is expected to be accomplished by developing estimation, diagnosis and reconfigurable methods that will enable extending for as long as possible the G\&C functionalities designed to assist the pilot in keeping the flight safe and making the flight task easier and the mission optimal. This goal is visualized by the shift seen in the bottom of Fig.10 from the dashed (current) to the solid line (the desire innovation).

This project is presented in detail in [Goupil et al 14], and can be followed at http://reconfigure.deimos-space.com/, thus no further details are given here in the interest of brevity except to mention that integrated approaches and high-performance architectures based on $\mathrm{H}_{\infty}$ optimization [Marcos and Balas 05b, Marcos et al 07] are candidate techniques being considered based on the successful consolidation of the technique from the previous cases.

\section{CONCLUSIONS}

This article has presented the efforts done at Deimos Space to take an established control technique to the field of aircraft fault detection and isolation from theory to practice. The technique, $\mathrm{H}_{\infty}$ optimization, has been shown to be capable of tackling a diverse array of faults (from actuator to sensor) for several types of commercial aircraft. All the cases presented, except for the $1^{\text {st }}$ one, were carried out during the past 7 years at Deimos Space (Spain) in the frame of European Union and European Space Agency efforts to consolidate the modelbased FDI technology in Europe. 


\section{REFERENCES}

Appleby B.D. (1990). Robust estimator design using the $\mathrm{H}_{\infty}$ norm and mu-synthesis. Ph.D. thesis, Department of Aeronautics and Astronautics, MIT.

Balas G.J., Doyle J.C., Glover K., Packard A., Smith R. (1998) Mu-Analysis and Synthesis Toolbox. The Mathworks Inc.

Chen J. and Patton R.J. (1999) Robust Model-based Fault Diagnosis for Dynamic Systems, Kluwer Academic Publishers: Dordrecht

Doyle J.C, Glover K., Khargonekar P.P., Francis B.A. (1988) State-space solutions to standard $\mathrm{H}_{2}$ and $\mathrm{H}_{\infty}$ control problems, IEEE Transactions Automatic Control, Vol AC-34, No.8, pp 831-847.

Edelmayer A., Bokor J., Keviczky L. (1994). An $\mathrm{H}_{\infty}$ filtering approach to robust detection of failures in dynamical systems. IEEE conference on decision and control, USA

Goupil, P., (2010) "Oscillatory Failure Case detection in the A380 Electrical Flight Control System by analytical redundancy," Control Engineering Practice, Vol.18, No.9, pp 1110-1119

Goupil P. and Marcos A. (2011) Advanced Diagnosis for Sustainable Flight Guidance and Control: The European ADDSAFE Project. SAE Technical Paper 2011-01-2804

Goupil P. and Marcos A. (2012) Industrial benchmarking and evaluation of ADDSAFE FDD designs. 8th IFAC SAFEPROCESS, Mexico

Goupil P., Boada-Bauxell J., Marcos A., Cortet E., Murray K., Costa H. (2014) AIRBUS efforts towards advanced real-time fault diagnosis and fault tolerant control, Submitted to IFAC World Congress

Hanke C., Nordwall D. (1970). The simulation of a jumbo jet transport aircraft. Vol. II: Modeling data. Technical report, NASA CR-114494, The Boeing Company.

Hanke C. (1971). The simulation of a large jet transport aircraft. Vol. I: Mathematical model. Technical report, NASA CR-1756, The Boeing Company.

Kerr M.L., Marcos A., Penin L.F. and Bornschlegl, E.(2008) "Gain Scheduled FDI for a Re-entry Vehicle", AIAA Guidance, Navigation and Control Conference, USA

Kerr M.L., Marcos A., Penin L.F., Briege O., Postlethwaite I., and Turner M. (2009) Piloted assessment of a fault diagnosis algorithm on the ATTAS aircraft. AIAA Guidance, Navigation and Control Conference, USA

Mangoubi, R. (1998) Robust estimation and failure detection: a concise treatment. Springer-Verlag

Marcos A., Ganguli S., Balas G.J. (2005a) An application of $\mathrm{H}_{\infty}$ fault detection and isolation to a transport aircraft. Control Engineering Practice, 13(1), 105-119

Marcos A. and Balas G.J. (2005b) A robust integrated controller/diagnosis aircraft application, International Journal of Robust and Nonlinear Control, 15:531-551

Marcos A., Turner M.C. and Postlethwaite I. (2007) An architecture for design and analysis of high-performance robust anti-windup compensators, IEEE Transactions on Automatic Control 52(9):1672-1679

Marcos A (2012) Assessment on the ADDSAFE Benchmark Simulator of an $\mathrm{H}_{\infty}$ Fault Detection Design for Aircraft. 8th IFAC SAFEPROCESS, Mexico
Marcos A. (2013) ADDSAFE Final Report, TN-D0.1.4-2, http://cordis.europa.eu/result/report/rcn/57197_en.html

Marzat, J., Piet-Lahanier H., Damongeot F., and Walter E. (2012) Model-based fault diagnosis for aerospace systems: a survey. Proceedings of the Institution of Mechanical Engineers, Part G: Journal of Aerospace Engineering, vol. 226 no. 10, pp 1329-1360

Melody J.W., Hillbrand T., Basar T., and Perkins W.R. (2001) $\mathrm{H}_{\infty}$ parameter identification for inflight detection of aircraft icing: The time-varying case. Control Engineering Practice, 9(12), 1327-1335

Niemann H. and Stoustrup J. (1997) Robust Fault Detection in Open Loop vs. Closed Loop. Proceedings of the 36th IEEE Conference on Decision and Control 